PROCEEDINGS OF THE

AMERICAN MATHEMATICAL SOCIETY

Volume 125, Number 12, December 1997, Pages 3465-3470

S 0002-9939(97)04263-9

\title{
CYCLIC BY PRIME FIXED POINT FREE ACTION
}

\author{
ALEXANDRE TURULL
}

(Communicated by Ronald M. Solomon)

\begin{abstract}
Let the finite group $A$ be acting on a (solvable) group $G$ and suppose that no non-trivial element of $G$ is fixed under the action of all the elements of $A$. Assume furthermore that $(|A|,|G|)=1$. A long standing conjecture is that then the Fitting height of $G$ is bounded by the length of the longest chain of subgroups of $A$. Even though this conjecture is known to hold for large classes of groups $A$, it is still unknown for some relatively uncomplicated groups. In the present paper we prove the conjecture for all finite groups $A$ that have a normal cyclic subgroup of square free order and prime index. Since many of these groups have natural modules where they act faithfully and coprimely but without regular orbits, the result is new for many of the groups we consider.
\end{abstract}

\section{INTRODUCTION}

Let $A$ be a finite group and assume that it acts as automorphisms of the finite group $G$ in such a way that the centralizer $\mathrm{C}_{G}(A)$ of the action of $A$ on $G$ is trivial $\mathrm{C}_{G}(A)=1$. Assume furthermore that $(|A|,|G|)=1$. It follows easily from the classification of finite simple groups that this implies that the group $G$ is solvable. For any solvable group $G$, we denote by $\mathrm{h}(G)$ its Fitting height. For any finite group $A$, we denote by $\ell(A)$ the length of the longest chain of subgroups of $A$. In the case when $A$ is solvable, $\ell(A)$ is simply the number of prime divisors of $|A|$ counting multiplicities, a fact that can be written, using the standard number theoretical function $\Omega$, as $\ell(A)=\Omega(|A|)$. In general, $\ell(A) \leq \Omega(|A|)$. A long standing conjecture is the following.

Conjecture. Let $A$ be any finite group and suppose it acts on the finite (solvable) group $G$ with $(|A|,|G|)=1$ and $\mathrm{C}_{G}(A)=1$. Then $\mathrm{h}(G) \leq \ell(A)$.

This inequality, if true, is known to be the best possible in a very strong sense (see [5]). If $B$ is any group and it acts on a group $S$, we say that it has a regular orbit if there is some element $v \in S$ such that

$$
\mathrm{C}_{B}(v)=\mathrm{C}_{B}(S) \text {. }
$$

Notice that, with this definition, $B$ need not act faithfully on $S$ to have a regular orbit. In [4], the conjecture is proved in the case in which, for every proper subgroup $B$ of $A$ and every $B$-invariant elementary abelian section $S$ of $G, B$ has a regular

Received by the editors June 11, 1996.

1991 Mathematics Subject Classification. Primary 20D45.

Key words and phrases. Solvable groups, fixed point free action, finite groups, representations. Partially supported by a grant from the NSF. 
orbit on $S$. Even though this result applies to many finite groups $A$, there are some uncomplicated groups to which it does not fully apply. For example, if $q$ is any power of a prime, and $r$ is a prime, there exists a semidirect product of a cyclic group of order $r$ and a cyclic group of order $\frac{q^{r}-1}{q-1}$ and a faithful module of order $q^{r}$ for it, upon which the semidirect product does not have any regular orbits. Some results that describe which semidirect products have regular orbits in primitive modules are obtained in [2]. Hence, the existence of regular orbits is far from guaranteed for acting groups $A$ which have a normal cyclic subgroup of prime index.

In [6], we generalized the theorem in [4] by proving that the conclusion still holds when we assume that regular orbits exist for only certain crucial sections. In the present paper, we use the results in [6] to prove the following result.

Theorem. Let $A$ be any finite group and suppose it acts on the finite (solvable) group $G$ with $(|A|,|G|)=1$ and $\mathrm{C}_{G}(A)=1$. Assume, furthermore, that $A$ has a normal cyclic subgroup of square free order and prime index. Then $\mathrm{h}(G) \leq \ell(A)$.

In 1978, A. D. Feldman [1] considered a similar problem, and obtained the inequality under the assumption that certain sections are not involved in $A$. This result was later superseded by the more general result of [4].

\section{NOTATION AND REMINDERS}

In this section we give the exact statement of the main result of [6], which we will use. We first need to fix some notation. We follow the notation of [6]. If $G$ is any finite group, we denote by $\mathrm{F}(G)$ the Fitting subgroup of $G$. We define $\mathrm{F}_{0}(G)=1$ and define inductively $\mathrm{F}_{i}(G)$ by $\mathrm{F}_{i}(G) / \mathrm{F}_{i-1}(G)=\mathrm{F}\left(G / \mathrm{F}_{i-1}(G)\right)$ for $i=1,2, \ldots$. If $G$ is solvable, the Fitting height $\mathrm{h}(G)$ of $G$ is the smallest non-negative integer $h$ such that $\mathrm{F}_{h}(G)=G$.

Hypothesis 1.1. $A$ and $G$ are finite groups, $G$ is solvable, and $A$ acts on $G$ by automorphisms. Furthermore, $(|A|,|G|)=1$.

Definition 1.2. Assume that $G$ is a finite solvable group. We define the top Fitting factor of $G$ to be 1 if $G=1$, and to be $G / \mathrm{F}_{\mathrm{h}(G)-1}(G)$ if $G \neq 1$. A section of the top Fitting factor of $G \neq 1$ is a section $S=H_{1} / H_{2}$, such that $H_{2} \supseteq \mathrm{F}_{\mathrm{h}(G)-1}(G)$.

Definition 1.3. Assume that $G$ is a finite group and that $S=H_{1} / H_{2}$ is a section of $G$, and let $G_{0}$ be any subgroup of $G$. We say that $G_{0}$ normalizes $S$ if it normalizes both $H_{1}$ and $H_{2}$. We further say that $G_{0}$ centralizes $S$ if it normalizes $S$ and $\left[G_{0}, H_{1}\right] \subseteq H_{2}$.

Definition 1.4. Assume Hypothesis 1.1. Then we define the set

$$
\begin{array}{r}
\mathcal{S}(A, G)=\{B \leq A: B \quad \text { centralizes some non-trivial elementary abelian } \\
\text { section of the top Fitting factor of } G\} .
\end{array}
$$

In particular, then, $\mathcal{S}(A, G)=\emptyset$ if $G=1$, and $1 \in \mathcal{S}(A, G)$ if $G \neq 1$. We are now ready to define the set $\mathcal{M}(B, G)$.

Definition 1.5. Assume Hypothesis 1.1. Let $B$ be any subgroup of $A$. We define $\mathcal{M}(B, G)$ as follows. 
(1) Suppose that $B \notin \mathcal{S}(A, G)$. Then $\mathcal{M}(B, G)$ consists of all the $B$-invariant elementary abelian sections of the top Fitting factor of $G$, together with all the $B$-invariant elementary abelian sections $S$ of $G$ that have the property that there exists some $B$-invariant subgroup $G_{0}$ of $G$ such that $G_{0}$ normalizes $S$, with $h=\mathrm{h}(G),\left(G_{0} \cap \mathrm{F}_{h-1}(G)\right) \mathrm{C}_{G_{0}}(S) \neq G_{0}$, and $B G_{0}$ acts irreducibly on $S$.

(2) Suppose that $B \in \mathcal{S}(A, G)$. Then $\mathcal{M}(B, G)$ consists of all the $B$-invariant elementary abelian sections of $G$.

With this notation we can state the main result of [6], which we will use to prove our theorem.

Theorem 1.6 ([6]). Let $A$ be any finite group and suppose it acts on the finite (solvable) group $G$ with $(|A|,|G|)=1$ and $\mathrm{C}_{G}(A)=1$. Assume, furthermore, that, for every proper subgroup $B$ of $A$ and every $S \in \mathcal{M}(B, G), B$ acts on the elementary abelian group $S$ with a regular orbit. Then $\mathrm{h}(G) \leq \ell(A)$.

\section{REGULAR ORBITS}

In this section we prove the results that will allow us to show (in the next section) that in a minimal counterexample to the theorem, $B$ will have regular orbits on all $S \in \mathcal{M}(B, G)$. For convenience, we will use $A$ instead of $B$ and will denote by $B$ the cyclic normal subgroup of $A$ of prime index.

Theorem 2.1. Suppose $A G$ is a finite group, $G \unlhd A G,(|A|,|G|)=1$ and $B$ is a cyclic normal subgroup of prime index of $A$. Furthermore, assume that $\mathrm{C}_{G}(B)=G$ but $[A, G]=G \neq 1$. Suppose $M$ is an $A G$-module over a field $F$ with characteristic $p, p$ not dividing $|A|$, faithful for $B G$. Then there exists some $v \in M$ such that $\mathrm{C}_{A}(v)=1$.

Proof. Suppose false. Choose a counterexample with minimum $|A G M|$. We split the proof into a number of steps.

Step 1. A is not abelian.

Proof. Let $K=\operatorname{ker}(M)$. Then $[K \cap A, G] \subseteq K \cap G=1$, so that $K \cap A \subseteq B$. But by hypothesis, $K \cap B=1$. It follows that $\operatorname{Res}_{A}^{A G}(M)$ is faithful. Now it is well known that if $A$ is abelian, this implies that $A$ has a regular orbit on $M$. This contradiction shows Step 1.

Step 2. $M$ is indecomposable.

Proof. Suppose not. Then $M=M_{1} \oplus M_{2}$, where $M_{1}$ and $M_{2}$ are non-trivial $A G$ modules. $G$ must act non-trivially on at least one of $M_{1}$ and $M_{2}$, say on $M_{1}$. By induction, there exists some $v_{1} \in M_{1}$ such that $\mathrm{C}_{A}\left(v_{1}\right)=\mathrm{C}_{B}\left(M_{1}\right)$. Since $B$ is cyclic, there exists some $v_{2} \in M_{2}$ such that $\mathrm{C}_{B}\left(v_{2}\right)=\mathrm{C}_{B}\left(M_{2}\right)$. Set $v=v_{1}+v_{2}$. Then $\mathrm{C}_{A}(v)=\mathrm{C}_{A}\left(v_{1}\right) \cap \mathrm{C}_{A}\left(v_{2}\right) \subseteq B$ since $\mathrm{C}_{A}\left(v_{1}\right) \subseteq B$. Hence,

$$
\mathrm{C}_{A}(v)=\mathrm{C}_{B}\left(v_{1}\right) \cap \mathrm{C}_{B}\left(v_{2}\right)=\mathrm{C}_{B}\left(M_{1}\right) \cap \mathrm{C}_{B}\left(M_{2}\right)=\mathrm{C}_{B}(M)=1 .
$$

The existence of $v$ then contradicts the fact that we are dealing with a counterexample to the theorem.

Step 3. $\operatorname{Res}_{A}^{A G}(M)$ is irreducible. 
Proof. $B \triangleleft A G$ and $\operatorname{Res}_{B}^{A G}(M)$ is the direct sum of its homogeneous components, because $p$ does not divide $|B|$. $A G$ permutes the different homogeneous components and, since $M$ is indecomposable by Step 2, it follows that $A G$ acts transitively on the homogeneous components of $\operatorname{Res}_{B}^{A G}(M)$. Hence, the kernels of all the irreducible submodules of $\operatorname{Res}_{B}^{A G}(M)$ are all conjugate in $A G$. Since $B$ is cyclic, this implies that they are all equal. Since $M$ is faithful, it follows that each irreducible submodule of $\operatorname{Res}_{B}^{A G}(M)$ is faithful.

Suppose $\operatorname{Res}_{A}^{A G}(M)=M_{1} \oplus M_{2}$, where $M_{1}$ and $M_{2}$ are proper submodules of $\operatorname{Res}_{A}^{A G}(M)$. There exist $v_{1} \in M_{1}$ and $v_{2} \in M_{2}$ such that $v_{1} \neq 0 \neq v_{2}$. It follows that $\mathrm{C}_{A}\left(v_{1}\right) \cap B=1=\mathrm{C}_{A}\left(v_{2}\right) \cap B$, because $B$ is cyclic and no non-trivial element of $B$ centralizes a non-trivial element of $M$. Since there is no regular orbit for $A$, we must have $\mathrm{C}_{A}\left(v_{1}\right) \neq 1 \neq \mathrm{C}_{A}\left(v_{2}\right)$. It follows that

$$
\left|\mathrm{C}_{A}\left(v_{1}\right)\right|=[A: B]=\left|\mathrm{C}_{A}\left(v_{2}\right)\right|
$$

are all prime, and $A=\mathrm{C}_{A}\left(v_{2}\right) B$. If $\mathrm{C}_{A}\left(v_{2}\right) \unlhd A$, then $\left[\mathrm{C}_{A}\left(v_{2}\right), B\right] \subseteq \mathrm{C}_{A}\left(v_{2}\right) \cap B=$ 1 , which implies that $A$ is abelian, against Step 1 . Hence $\mathrm{C}_{A}\left(v_{2}\right) \not A A$ and there exists some $a \in A$ such that $\mathrm{C}_{A}\left(v_{1}\right) \neq \mathrm{C}_{A}\left(v_{2}\right)^{a}$. Set $v=v_{1}+a^{-1} v_{2}$. Then

$$
\mathrm{C}_{A}(v)=\mathrm{C}_{A}\left(v_{1}\right) \cap \mathrm{C}_{A}\left(a^{-1} v_{2}\right)=\mathrm{C}_{A}\left(v_{1}\right) \cap \mathrm{C}_{A}\left(v_{2}\right)^{a}=1,
$$

because $\left|\mathrm{C}_{A}\left(v_{1}\right)\right|=\left|\mathrm{C}_{A}\left(v_{2}\right)^{a}\right|$ is a prime. This is a contradiction. Hence Step 3 holds.

Step 4. $\operatorname{Res}_{B}^{A G}(M)$ is irreducible.

Proof. Suppose first that $\operatorname{Res}_{B}^{A G}(M)$ is not homogeneous. Then, as $\operatorname{Res}_{A}^{A G}(M)$ is irreducible and $[A: B]$ is a prime, $\operatorname{Res}_{B}^{A G}(M)$ is the direct sum of $[A: B]$ different homogeneous components. Let

$$
\operatorname{Res}_{B}^{A G}(M)=N_{1} \oplus \cdots \oplus N_{\alpha}
$$

be the decomposition of $\operatorname{Res}_{B}^{A G}(M)$ into homogeneous components. Then $\alpha=$ $[A: B]$. Let $v \in N_{1}, v \neq 0$. Then $\mathrm{C}_{A}(v) \subseteq B$ because $A / B$ acts regularly on the set $\left\{N_{1}, \ldots, N_{\alpha}\right\}$. But no non-trivial element of $B$ centralizes a non-trivial element of $M$. Hence, $\mathrm{C}_{A}(v)=1$, a contradiction. Hence, $\operatorname{Res}_{B}^{A G}(M)$ is homogeneous.

Let $K \supseteq F$ be a splitting field for $A$ and $B$. Then $\operatorname{Res}_{A}^{A G}(K \otimes M)$ is the direct sum of non-isomorphic irreducible $K A$-modules, because $\operatorname{Res}_{A}^{A G}(M)$ is irreducible and the characteristic of $K$ is finite. Since the absolutely irreducible faithful $K A$ modules restrict to $K B$-modules as a direct sum of $[A: B]$ non-isomorphic $K B$ modules, and non-isomorphic $K A$-modules restrict disjointly to sets of irreducible $K B$-modules, $\operatorname{Res}_{B}^{A G}(K \otimes M)$ is the direct sum non-isomorphic $B$-irreducible modules. It follows, since $\operatorname{Res}_{B}^{A G}(M)$ is homogeneous, that $\operatorname{Res}_{B}^{A G}(M)$ is irreducible, as desired.

Step 5. The theorem holds.

Proof. The existence of $v$ now follows immediately from [2, Proposition 2.1 and Proposition 1.4], which yield the final contradiction. The existence of $v$ can also be shown more directly as follows. By Step 4, the image in $\operatorname{End}_{F}(M)$ of $F B$ is a field $K$. By Schur's Lemma, $\mathrm{C}_{\operatorname{End}_{F}(M)}(K)=K$. Since $G$ centralizes $B$, it follows that the image of $B$ and that of $G$ are subgroups of $K^{\times}$. Let $v_{0} \in M, v_{0} \neq 0$. Since by hypothesis $\mathrm{C}_{A}(v) \neq 1$ for all $v \in M$ and $\mathrm{C}_{B G}\left(v_{0}\right)=1$, it follows that $\mathrm{C}_{A G}\left(v_{0}\right)$ is contained in $A$ and has prime order equal to $[A: B]$. Now since $[A, G]=G \neq 1$, the 
structure of $A G / B$ implies that there exists some $g \in G$ such that $\mathrm{C}_{A G}\left(v_{0}\right)^{g} \nsubseteq \nexists A$. It then follows that, setting $v=g^{-1} v_{0}$, we have $\mathrm{C}_{A}(v)=\mathrm{C}_{A G}\left(v_{0}\right)^{g} \cap A=1$. This contradicts the fact that we are dealing with a counterexample to the theorem.

Corollary 2.2. Suppose $A G$ is a finite group, $G \triangleleft A G,(|A|,|G|)=1$ and $B$ is a cyclic normal subgroup of prime index of $A$. Further assume that $[B, G] \subseteq K$ and $\mathrm{C}_{G / K}(A)=1$, where $K$ is a normal subgroup of $A G$ properly contained in $G$. Let $M$ be some $F A G$-module, faithful for $B G$, where $F$ is a field of characteristic $p, p$ not dividing $|A|$. Then there exists some $v \in M$ such that $\mathrm{C}_{A}(v)=1$.

Proof. Since $G / K \neq 1$, there exists some prime $q$ such that $q$ divides $|G / K|$. Let $Q$ be an $A$-invariant Sylow $q$-subgroup of $G$. Since $B$ acts trivially on $G / K, B$ centralizes $Q / Q \cap K$, so that $\mathrm{C}_{Q}(B) \nsubseteq K$. Since $\mathrm{C}_{G / K}(A)=1$, it follows that $\left[A, \mathrm{C}_{Q}(B)\right] \neq 1$. Set $\left[A, \mathrm{C}_{Q}(B)\right]=Q_{0}$. Now $B$ centralizes $Q_{0}$ and $\left[A, Q_{0}\right]=Q_{0}$. Hence the corollary follows immediately from Theorem 2.1.

\section{THE MAIN THEOREMS}

In this section we prove the main theorems. If we are willing to add some hypotheses on the action of $A$ on $G$, we can allow more general groups $A$. In particular we can take $A$ to be any finite group with a cyclic normal subgroup of prime index.

Theorem 3.1. Let $A$ be a finite group and suppose it acts on the finite (solvable) group $G$ with $(|A|,|G|)=1$ and $\mathrm{C}_{G}(A)=1$. Assume, furthermore, that $A$ has a normal abelian subgroup $C$ of prime index such that $C$ centralizes the top Fitting factor of $G$ and for each proper non-abelian subgroup $B$ of $A$ we have that $B \cap C$ is cyclic. Then $\mathrm{h}(G) \leq \ell(A)$.

Proof. Assume the theorem is false. Then $G \neq 1$. By Definition 1.4, $\mathcal{S}(A, G)$ is the set of all the subgroups of $C$. Let $B$ be any proper subgroup of $A$ and let $S \in \mathcal{M}(B, G)$. If $B$ is abelian, then $B$ acts on $S$ with regular orbits. Hence, assume that $B$ is not abelian. By hypothesis, it now follows that $B$ has a normal cyclic subgroup $B \cap C$ of prime index. If $B \in \mathcal{S}(A, G)$, then $B$ is contained in $C$ and therefore $B$ is abelian. Hence, $B \notin \mathcal{S}(A, G)$. If $S$ is a section of the top Fitting factor of $G$, since $A / C$ is cyclic and $C$ acts trivially on the top Fitting factor of $G$, it follows that $B$ acts on $S$ with regular orbits again in this case. Hence, assume that $S$ is not a section of the top Fitting factor of $G$. From Definition 1.5, it follows that there exists some $B$-invariant subgroup $G_{0}$ of $G$ such that $G_{0}$ normalizes $S$, with $h=\mathrm{h}(G),\left(G_{0} \cap \mathrm{F}_{h-1}(G)\right) \mathrm{C}_{G_{0}}(S) \neq G_{0}$, and $B G_{0}$ acts irreducibly on $S$. Set $K=\left(G_{0} \cap \mathrm{F}_{h-1}(G)\right) \mathrm{C}_{G_{0}}(S)$. Then $K$ is a proper subgroup of $G_{0}$ and it is normal in $B G_{0}$. Since $A$ acts fixed point freely on $G$ and $C$ acts trivially on the top Fitting factor of $G$, it follows that any subgroup of $A$ not contained in $C$ is acting fixed point freely on the top Fitting factor of $G$. In particular, since $B \notin \mathcal{S}(A, G), B$ acts fixed point freely on the the top Fitting factor of $G$. It follows that $B$ is acting fixed point freely on $G_{0} / K$. Hence, by Corollary $2.2, B$ acts on $S$ with regular orbits. Hence, we have shown that for all proper subgroup $B$ of $A$ and all $S \in \mathcal{M}(B, G)$, $B$ acts on $S$ with regular orbits. Hence, Theorem 1.6 can be applied, and it yields that $\mathrm{h}(G) \leq \ell(A)$, as desired.

Finally, we prove the theorem mentioned in the introduction. 
Theorem 3.2. Let $A$ be any finite group and suppose it acts on the finite (solvable) group $G$ with $(|A|,|G|)=1$ and $\mathrm{C}_{G}(A)=1$. Assume, furthermore, that $A$ has a normal cyclic subgroup $C$ of square free order and prime index. Then $\mathrm{h}(G) \leq \ell(A)$.

Proof. Assume the theorem is false. Among all counterexamples select one with $|A G|$ as small as possible. We say [3] that a sequence of $A$-invariant subgroups of $G$ $\left(\hat{P}_{i}\right), i=1, \ldots, h$, is an irreducible $A$-tower of height $h$ if the following are satisfied:

(1) $\hat{P}_{i}$ is a $p_{i}$-group $\left(p_{i}\right.$ some prime) for $i=1, \ldots, h$;

(2) $\hat{P}_{i}$ normalizes $\hat{P}_{j}$, for $i<j$;

(3) We set $P_{h}=\hat{P}_{h}$ and $P_{i}=\hat{P}_{i} / \mathrm{C}_{\hat{P}_{i}}\left(P_{i+1}\right)$ for $i=1, \ldots, h-1$, and $P_{1} \neq 1$.

(4) $p_{i} \neq p_{i+1}$ for $i=1, \ldots, h-1$.

(5) $\phi\left(\phi\left(P_{i}\right)\right)=1, \phi\left(P_{i}\right) \subseteq \mathrm{Z}\left(P_{i}\right)$ and, if $p_{i} \neq 2, \exp \left(P_{i}\right)=p_{i}$ for $i=1, \ldots, h$ and $\hat{P}_{i-1}$ centralizes $\phi\left(P_{i}\right)$ for $i=2, \ldots, h$;

(6) $P_{1}$ is elementary abelian;

(7) There exists $H_{i}$ an elementary abelian subgroup of $P_{i-1}$ normalized by $A$ such that $\left[H_{i}, P_{i}\right]=P_{i}$ for $i=2, \ldots, h$;

(8) If $Q \subseteq \hat{P}_{i}$ for some $i, Q$ is normalized by $A \hat{P}_{1} \cdots \hat{P}_{i-1}$ and its image in $P_{i}$ is not contained in $\phi\left(P_{i}\right)$, then $Q=\hat{P}_{i}$.

By [3, Lemma 1.9], $G$ does contain an irreducible $A$-tower of height $h=\mathrm{h}(G)$. By the minimality, since $\mathrm{h}\left(\hat{P}_{1} \cdots \hat{P}_{h}\right)=h$, it follows that $G=\hat{P}_{1} \cdots \hat{P}_{h}$.

Let $B$ be any subgroup of prime order of $C$. Suppose $B$ does not centralize $P_{1}$. Then by the proof of [3, Theorem 3.1], $\left(\mathrm{C}_{\hat{P}_{i}}(B)\right)$ for $i=2, \ldots, h$ satisfies Conditions 1, 2 and 3. By Condition 6 and Condition 7, this proof amounts to a repeated application of [3, Theorem $2.1(\mathrm{~A})]$. Since $B$ is acting non-trivially on $P_{1}$, and $P_{1}$ is abelian, then the centralizer of $B$ in the appropriate section of $P_{2}$ is large enough to apply [3, Theorem 2.1 (A)] again, this time considering the action of $P_{2}$ on $P_{3}$, etc. Since $\left(\mathrm{C}_{\hat{P}_{i}}(B)\right)$ for $i=2, \ldots, h$ also satisfies Condition 4, it follows that $\mathrm{h}\left(\mathrm{C}_{G}(B)\right) \geq h-1$, for it contains a tower of height $h-1$ (see $[3])$. Since $A / B$ is acting fixed point freely on $\mathrm{C}_{G}(B)$, it follows by induction that $h-1 \leq \mathrm{h}\left(\mathrm{C}_{G}(B)\right) \leq \ell(A)-1$. This implies the desired inequality. Therefore, every subgroup of $C$ of prime order acts trivially on $P_{1}$. Since $C$ has square free order, it follows that $C$ centralizes $P_{1}$.

Now $P_{1}$ is isomorphic to the top Fitting factor of $G$. Hence, the result follows immediately from Theorem 3.1.

\section{REFERENCES}

1. A.D. Feldman, Fitting height of solvable groups admitting fixed-point-free automorphism groups, J. Algebra 53 (1978), 268 - 295. MR 58:5903

2. A. Turull, Supersolvable automorphism groups of solvable groups, Math. Z. 183 (1983), 47 73. MR 85d:20030

3. A. Turull, Fitting height of groups and of fixed points, J. Algebra 86 (1984), 555 - 566. MR 85i:20021

4. A. Turull, Fixed point free action with regular orbits, J. reine angew. Math. 371 (1986), 67 91. MR 88c:20029

5. A. Turull, Generic fixed point free action of arbitrary finite groups, Math. Z. 187 (1984), 491 - 503. MR 86a:20020

6. A. Turull, Fixed point free action with some regular orbits, J. Algebra (to appear).

Department of Mathematics, University of Florida, Gainesville, Florida 32611

E-mail address: turull@math.ufl.edu 